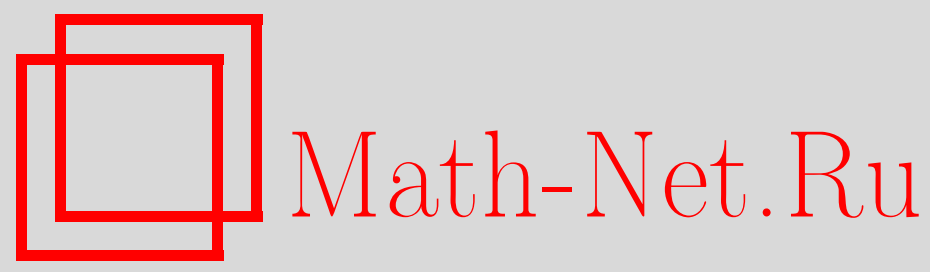

P. А. Шарипов, Е. Н. Цыганов, О сепаратной алгебраичности вдоль семейств алгебраических кривых, Матем. заметки, 2000, том 68, выпуск 2, 294-302

DOI: https://doi.org/10.4213/mzm946

Использование Общероссийского математического портала Math-Net.Ru подразумевает, что вы прочитали и согласны с пользовательским соглашением http://www.mathnet.ru/rus/agreement

Параметры загрузки:

IP : 3.85 .73 .92

26 апреля 2023 г., 15:51:27

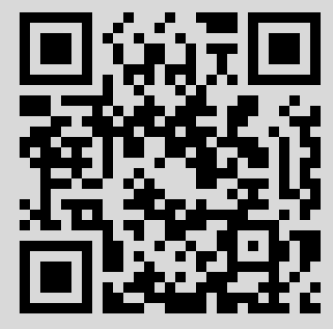




\title{
О СЕПАРАТНОЙ АЛГЕБРАИЧНОСТИ ВДОЛЬ СЕМЕЙСТВ АЛГЕБРАИЧЕСКИХ КРИВЫХ
}

\author{
Е. Н. Цыганов, Р. А. Шарипов
}

В статье приводятся одно обобщение классической теоремы об алгебраичности по отдельным переменньм и последующее ее доказательство.

Библиографоия: 17 названий.

1. Введение. Теорема о голоморфности по отдельным переменным (теорема Хартогса) является одной из фундаментальных теорем в теории функций многих комплексных переменных (см., например, [1]). Она формулируется следующим образом.

Теорема 1. Пусть функиия $f(z)=f\left(z_{1}, \ldots, z_{n}\right)$, определенная в области $D \subset \mathbb{C}^{n}$, является голоморфной по каждой переменной $z_{i}$ при любых фиксированных значениях остальных переменных. Тогда $f$ голоморфна в $D$.

Теорема 1 имеет определенную модификацию для полиномиальных, рациональных и алгебраических функций (см. [2]). В последнем случае мы имеем следующее утверждение.

ТЕОрема 2. Пусть голоморфная в области $D \subset \mathbb{C}^{n}$ функиия $f(z)$ алгебраична по каждой переменной $z_{i}$ при любых фиксированных значениях остальных переменных. Тогда она является голоморфной ветвью в $D$ для некоторой алгебраической функиии, заданной полиномиальным уравнением $P\left(f, z_{1}, \ldots, z_{n}\right)=0$.

В обоих случаях мы можем рассматривать следующие $(n-1)$-параметрические семейства комплексных прямых в $D$ :

$$
\begin{cases}z_{i}=c_{i}^{(m)}=\mathrm{const} & \text { для } i \neq m, \\ z_{m}=t \in \mathbb{C} & \text { для } i=m .\end{cases}
$$

Здесь $t \in \mathbb{C}$ - комплексный параметр на прямой, а константы $c_{i}^{(m)}$ параметризуют прямые в $m$-м семействе. При сужении $f(z)$ на любую прямую вида (1.1) мы получаем следующую функцию:

$$
f_{m}(t)=f\left(c_{1}^{(m)}, \ldots, c_{m-1}^{(m)}, t, c_{m+1}^{(m)}, \ldots, c_{n}^{(m)}\right),
$$

Работа вьполнена при финансовой поддержке Европейского фонда INTAS, грант № 93-47 (руководитель С. И. Пинчук), Российского фонда фундаментальных исследований, грант № 96-01-00127 (руководитель Я. Т. Султанаев), а также поддержана грантом АН Республики Башкортостан (руководитель Н. М. Асадуллин). 
которая голоморфна по $t$ в предположениях теоремы 1 и алгебраична по $t$ в предположениях теоремы 2.

В терминах координатньх прямых (1.1) вышеупомянутая классическая теорема 2 может быть сформулирована так: каждая голоморфная функция $f(z)$, которая алгебраична вдоль каждой из координатных прямых в $D$, есть алгебраическая функция многих комплексных переменных. В настоящей статье мы рассматриваем несколько обобщенных версий этого утверждения, когда координатные прямые замены определенными классами комплексных кривых. Необходимость такого обобщения была замечена А. Б. Суховьм [3], [4] в связи с проблемой Пуанкаре-Александера (см. [5]-[8]) и ее обобщениями (см. [9]-[13]).

В качестве первого шага в [3], [4] координатные прямые были заменены квадриками. Второй шаг был сделан в [14], [15]. Здесь вместо координатных прямых рассматриваются произвольные алгебраические кривые. Они взяты в параметрической форме с комплексным параметром $t \in \mathbb{C}$ :

$$
\left\{\begin{array}{c}
z_{1}=R_{1}^{(m)}\left(t, c_{1}^{(m)}, \ldots, c_{n-1}^{(m)}\right) \\
\ldots \ldots \ldots \ldots \ldots \ldots \ldots \ldots \ldots \\
z_{n}=R_{n}^{(m)}\left(t, c_{1}^{(m)}, \ldots, c_{n-1}^{(m)}\right) .
\end{array}\right.
$$

Алгебраические кривые (1.3) образуют $n$ семейств $R^{(1)}, \ldots, R^{(n)}$, индекс $m$ нумерует эти семейства. Координатные функции $R_{i}^{(m)}$ в $(1.3)$ алгебраичны по $t$. Выбор констант $c_{1}^{(m)}, \ldots, c_{n-1}^{(m)}$ фиксирует конкретную кривую в $m$-м семействе.

Заметим, что в общем случае все множество кривых (1.3) нельзя одновременно распрямить, превратив в координатные прямые при помощи алгебраического отображения. Поэтому для доказательства теорем, обобщающих теорему 2 , необходимо развитие специального апшарата.

Для заданной функции $f(z)$ в $D$ мы можем рассмотреть ее сужения на кривые (1.3). Это дает нам функции $f_{m}(t)$, аналогичные функциям (1.2):

$$
f_{m}(t)=f\left(R_{1}^{(m)}\left(t, c_{1}^{(m)}, \ldots, c_{n-1}^{(m)}\right), \ldots, R_{n}^{(m)}\left(t, c_{1}^{(m)}, \ldots, c_{n-1}^{(m)}\right)\right) .
$$

Скажем, что функция $f(z)$ алгебраична вдоль кривых (1.3), если любая функция $f_{m}(t)$ в (1.4) алгебраически зависит от параметра $t$.

ТЕОрема 3 (гипотеза ${ }^{1}$ ). Пусть голоморфная в области $D \subset \mathbb{C}^{n}$ функиия $f(z)$ алгебраична вдоль алгебраических кривых, образующих $n$ регулярны $x^{2}$ семейств в общем положении ${ }^{2}$. Тогда она является голоморфной ветвью в $D$ для некоторой алгебраической функиии, заданной полиномиальным уравнением $P\left(f, z_{1}, \ldots, z_{n}\right)=0$.

Данная теорема 3 была доказана в [14], [15], но в более слабой формулировке. Координатные функции $R_{i}^{(m)}$ в (1.3) предполагались алгебраическими не только по $t$, но и по всему множеству их аргументов $t, c_{1}^{(m)}, \ldots, c_{n-1}^{(m)}$. В настоящей статье мы исключаем это дополнительное предположение относительно алгебраической зависимости $R_{i}^{(m)}$ от

\footnotetext{
${ }^{1} Э$ та гипотеза хорошо известна в среде специалистов и, практически, никто не сомневается в ее справедливости. Авторам она стала известна от А. Б. Сухова.

${ }^{2}$ Точное определение см. в п. 2.
} 
$c_{1}^{(m)}, \ldots, c_{n-1}^{(m)}$, но делаем более сильное ограничение на зависимость $z_{i}$ и $f_{m}$ от основной переменной $t$ в (1.3) и (1.4). Функции $z_{1}(t), \ldots, z_{n}(t)$ и $f_{m}(t)$ выбираются полиномами по $t$. Ниже приведена формулировка основной теоремы, которую мы докажем в этой статье.

ТЕОремА 4. Пусть функиия $f(z)=f\left(z_{1}, \ldots, z_{n}\right)$ голоморфна в области $D \subset \mathbb{C}^{n}$ и является полиномом вдоль полиномиальных кривых, образующих $n$ регулярных семейств общего полохсения. Тогда $f(z)$ является голоморфной ветвью в $D$ некоторой алгебраической функиии, заданной полиномиальным уравнением

$$
P\left(f, z_{1}, \ldots, z_{n}\right)=0
$$

Самая общая теорема 3 все еще остается недоказанньм результатом. Ее доказательство будет предметом наших следующих публикаций.

Данная статья имеет следующую структуру. В п. 2 мы приводим точные определения. Пункт 3 содержит набросок доказательства центральной теоремы 4. Здесь мы опускаем детали. Пункты 4 и 5 посвящены строгому доказательству всех вспомогательных результатов, которые необходимы при доказательстве основной теоремы.

2. Определения и некоторые предварительные результаты. Параметрические уравнения (1.3) определяют базовые семейства полиномиальных кривых. Чтобы удовлетворять теореме 4, они должны быть регулярными в смысле следующего определения.

ОПРЕДЕЛЕНИЕ 1. Назовем $m$-е семейство полиномиальных кривых (1.3) peгулярным в области $D \subset \mathbb{C}^{n}$, если соответствующее отображение

$$
R^{(m)}:\left(t, c_{1}^{(m)}, \ldots, c_{n-1}^{(m)}\right) \mapsto\left(z_{1}, \ldots, z_{n}\right) \in D
$$

в (1.3) является биголоморфным диффеоморфизмом некоторой области $U_{m}$ из $\mathbb{C}^{n}$ в область $D$.

Если $m$-е семейство регулярно, то мы можем считать $t, c_{1}^{(m)}, \ldots, c_{n-1}^{(m)}$ комплексными криволинейньми координатами в $D$. Отображение

$$
\varphi_{\tau}^{(m)}:\left(t, c_{1}^{(m)}, \ldots, c_{n-1}^{(m)}\right) \mapsto\left(t+\tau, c_{1}^{(m)}, \ldots, c_{n-1}^{(m)}\right)
$$

есть перемещение вдоль кривой $m$-го семейства, а $\tau$ - параметр перемещения. С помощью диффеоморфизма (2.1) мы можем переписать (2.2) в декартовых координатах. Здесь это отображение $\varphi_{\tau}^{(m)}: z \mapsto \widetilde{z}$ задается следующим набором голоморфных функций

$$
\left\{\begin{array}{c}
\widetilde{z}_{1}=\varphi_{1}^{(m)}\left(\tau, z_{1}, \ldots, z_{n}\right) \\
\ldots \ldots \ldots \ldots \ldots \ldots \ldots \\
\widetilde{z}_{n}=\varphi_{n}^{(m)}\left(\tau, z_{1}, \ldots, z_{n}\right) .
\end{array}\right.
$$

Из $(2.2)$ мы находим, что $\varphi_{\tau}^{(m)}\left(\varphi_{\theta}^{(m)}(z)\right)=\varphi_{\tau+\theta}^{(m)}(z)$. Значит, $\varphi_{\tau}^{(m)}$ определяют голоморфную однопараметрическую локальную групу диффеоморфизмов в области $D$. Эта локальная группа порождает голоморфное векторное поле $\boldsymbol{X}_{m}$ в $D$ - поле касательных 
векторов к кривым $m$-го семейства. В предположениях теоремы 4 у нас есть $n$ голоморфных векторных полей:

$$
\boldsymbol{X}_{1}, \ldots, \boldsymbol{X}_{n}
$$

В криволинейных координатах $t, c_{1}^{(m)}, \ldots, c_{n-1}^{(m)} m$-е векторное поле $\boldsymbol{X}_{m}$ из $(2.4)$ распрямляется и изображается дифференциальным оператором

$$
\boldsymbol{X}_{m}=\frac{\partial}{\partial t}
$$

Однако, криволинейных координат, в которых распрямлялись бы одновременно все векторные поля (2.4), в общем случае не существует, ибо векторные поля $\boldsymbol{X}_{1}, \ldots, \boldsymbol{X}_{n}$ в общем случае не являются коммутируюшими.

ОПРЕДЕЛЕНИЕ 2. Если задано $n$ регулярных семейств полиномиальных кривых в области $D \subset \mathbb{C}^{n}$, то будем говорить, что они находятся в общем положении, если соответствующие векторные поля (2.4) линейно независимы в каждой точке $z \in D$.

Теперь рассмотрим полиномиальные кривые (1.3), которые по предположению теоремы 4 образуют $n$ регулярных семейств общего положения. Обозначим через $d_{1 m}, \ldots$, $d_{n m}$ и $p_{m}$ степени полиномов $z_{1}(t), \ldots, z_{n}(t)$ и $f_{m}(t)$ в $(1.3)$ и (1.4). Они зависят от выбора констант $c_{1}^{(m)}, \ldots, c_{n-1}^{(m)}$. Поскольку $m$-е семейство кривых регулярно в области $D$, мы можем считать $d_{1 m}, \ldots, d_{n m}$ и $p_{m}$ зависящими от точки $z \in D$ :

$$
\begin{array}{rlrl}
d_{i m}(z) & =\operatorname{deg} R_{i}^{(m)}(t), \quad i & =1, \ldots, n, \quad m=1, \ldots, n, \\
p_{m}(z) & =\operatorname{deg} f_{m}(t), \quad m=1, \ldots, n .
\end{array}
$$

Функции (2.6) - это целочисленные функции в $D$, их значения остаются постоянными вдоль кривых соответствующего семейства. В п. 4 мы докажем следующую теорему относительно функций (2.6).

ТеОрема 5. В предположениях теоремы 4 существует меньшая подобласть $\widetilde{D} \subset D$ такая, что все функиии (2.6) постоянны в $\widetilde{D}$.

Теперь мы можем изложить идею доказательства центральной теоремы 4.

3. Доказательство теоремы 4. Заметим, что в теореме 5 мы сжимаем область $D$ до $\widetilde{D} \subset D$. Это никак не влияет на окончательньй результат, так как голоморфная в $D$ функция $f(z)$, алгебраическая в некоторой меньшей подобласти $\widetilde{D}$, является алгебраической и в $D$. Поэтому мы можем уменьшить нашу область настолько, насколько это нам необходимо. Согласно теореме 5 у нас есть подобласть $\widetilde{D} \subset D$, где все степени полиномов в (1.3) и (1.4) постоянны: $\operatorname{deg} R_{i}^{(m)}(t)=d_{i m}, \operatorname{deg} f_{m}(t)=p_{m}$. Возьмем целые неотрицательные числа $k_{1}, \ldots, k_{n}$ и $q$ и построим моном:

$$
M\left(k_{1}, \ldots, k_{n}, q\right)=f^{q} \cdot\left(z_{1}\right)^{k_{1}} \cdots\left(z_{n}\right)^{k_{n}},
$$

где $f=f(z)$. Моном (3.1) является голоморфной функцией в области $\widetilde{D}$. Рассмотрим сужение этой функции на произвольную кривую $m$-го семейства в $\widetilde{D}$. Сужение монома $M\left(k_{1}, \ldots, k_{n}, q\right)$ есть полином по $t$, его степень выражается формулой

$$
q_{m}=\operatorname{deg} M\left(k_{1}, \ldots, k_{n}, q\right)=q p_{m}+\sum_{i=1}^{n} k_{i} d_{i m}
$$


Если $N>q_{m}$ - некоторое целое число, то, применяя $N$-ю степень дифференциального оператора (2.5) к моному (3.1), мы получаем

$$
\left(\boldsymbol{X}_{m}\right)^{N} M\left(k_{1}, \ldots, k_{n}, q\right)=0 .
$$

Относительно $M\left(k_{1}, \ldots, k_{n}, q\right)$ равенство (3.3) есть линейное дифференциальное уравнение $N$-го порядка с голоморфными коэффициентами. Теперь объединим все равенства (3.3) в систему.

$$
\left(\boldsymbol{X}_{m}\right)^{N} \varphi(z)=0, \quad m=1, \ldots, n,
$$

и определим $V(N)$ как множество, состоящее из решений системы уравнений $(3.4)$, голоморфных в области $\widetilde{D}$ :

$$
V(N)=\left\{\varphi \in \mathcal{H}(\widetilde{D}):\left(\boldsymbol{X}_{m}\right)^{N} \varphi=0, m=1, \ldots, n\right\} .
$$

Поскольку уравнения (3.4) линейны и однородны, множество их решений $V(N)$ образует линейное пространство над полем комплексных чисел.

Теорема 6. Для любого иелого $N>0$ комплексное линейное пространство (3.5) имеет конечную размерность $и \operatorname{dim} V(N)<(n N)^{n}$.

В п. 4 мы докажем эту теорему, дав оценку для $\operatorname{dim} V(N)$. Определим $M(N)$ как число мономов (3.1) таких, что степень (3.2) их сужения на кривые меньше, чем $N$, т.е. $q_{m}<N$ для всех $m=1, \ldots, n$. Если для некоторого значения $N$ мы найдем, что $M(N)>(n N)^{n}$, то мы получим, что некоторьй набор мономов линейно зависим над полем комплексных чисел $\mathbb{C}$. Это даст полиномиальное уравнение $P\left(f, z^{1}, \ldots, z_{n}\right)=0$ на функцию $f(z)$, что и будет доказательством теоремы 4. Поэтому заключительным шагом в доказательстве теоремы 4 должна быть требуемая оценка для $M(N)$ по крайней мере для какого-то одного значения $N$.

Обозначим $p=\max \left\{p_{1}, \ldots, p_{n}\right\}$ и $d_{i}=\max \left\{d_{i 1}, \ldots, d_{i n}\right\}$ (см. формулу (3.2) вьшш). Выберем произвольное целое число $K>1$ и положим

$$
N=N(K)=1+K p+\sum_{i=1}^{n} K d_{i} .
$$

Тогда для $q=1, \ldots, K$ и для $k_{i}=1, \ldots, K$ степени соответствующих мономов $(3.1)$ не превосходят $N$. Для числа же таких мономов отсюда находим, что $M(N) \geqslant K^{n+1}$. Для размерности пространства $V(N)$ из (3.6) и из теоремы 6 выводим

$$
\operatorname{dim} V(N) \leqslant(n N)^{n} \leqslant \text { const } \cdot K^{n}, \quad K \rightarrow \infty .
$$

Сравнивая полученные две оценки, заключаем, что $M(N)>\operatorname{dim} V(N)$ для некоторого достаточно большого значения $K$. Таким образом, основная теорема 4 доказана при условии справедливости теорем 5 и 6 . Оставшаяся часть статьи посвящена доказательству этих двух вспомогательных теорем.

4. Доказательство теоремы 5. Рассмотрим целочисленные функции $d_{i m}(z)$ и $p_{m}(z)$ из (2.6). Каждая из этих функций задает отображение из $D$ в множество целых чисел $\mathbb{Z}$, поэтому вся совокупность этих функций может трактоваться как отображение

$$
\nu: D \rightarrow \mathbb{Z}^{r}
$$

где $r=n(n+1)$. Здесь в $(4.1)$ множество $S=\mathbb{Z}^{r}$ является счетньм. Поэтому мы можем применить следующую теорему. 
Теорема 7. Для произвольного отображсения $\nu: D \rightarrow S$ из области $D$ в счетное множество $S$ мохно найти по крайней мере одно значение $s \in S$ такое, что полный прообраз $\nu^{-1}(s)=\{z \in D: \nu(z)=s\}$ есть мнохество, плотное $\boldsymbol{в}$ некоторой подобласти $\widetilde{D} \subset D$.

Мы не будем доказьвать эту топологическую теорему 7 , поскольку она является непосредственным следствием теоремы Бэра о категории (см. [16, гл. $3, \S 5$, теорема 8] и $[17$, гл. $2, \S 3])$. Аналогичные теоремы использовались в [2] и в [14], [15].

Фиксируем значение $s \in S$, полньй прообраз которого $\nu^{-1}(s)$ плотен в подобласти $\widetilde{D}$. Обозначим $F=\nu^{-1}(s) \cap \widetilde{D}$. Фиксация $s$ означает, что степени полиномов $(2.6)$ принимают одни и те же значения во всех точках множества $F$, плотного в $\widetilde{D}$. Пользуясь регулярностью $m$-го семейства кривых (см. определение 1 ), выберем криволинейные координаты $t, c_{1}^{(m)}, \ldots, c_{n-1}^{(m)}$ в области $\widetilde{D}$. Тогда условия $d_{i m}=\operatorname{deg} R_{i}^{(m)}(t)$ и $p_{m}(z)=\operatorname{deg} f_{m}(t)$ можно записать так:

$$
\begin{array}{ll}
\frac{\partial^{k} R_{i}^{(m)}}{\partial t^{k}} \neq 0 \quad \text { при } k=d_{i m}, & \frac{\partial^{k} f_{m}}{\partial t^{k}} \neq 0 \quad \text { при } k=p_{m}, \\
\frac{\partial^{k} R_{i}^{(m)}}{\partial t^{k}}=0 \text { при } k>d_{i m}, & \frac{\partial^{k} f_{m}}{\partial t^{k}}=0 \quad \text { при } k>p_{m} .
\end{array}
$$

Эти условия выполнены во всех точках множества $F$. Из зануления старших производных (4.3) в точках плотного множества $F$ вытекает их зануление во всех точках области $\widetilde{D}$. Поэтому $\operatorname{deg} R_{i}^{(m)}(t) \leqslant d_{i m}$ и $\operatorname{deg} f_{m}(t) \leqslant p_{m}$ в $\widetilde{D}$.

Рассмотрим некоторую одну точку $z_{0} \in F$. В ней вьполнены условия (4.2). В силу голоморфности $R_{i}^{(m)}$ и $f_{m}$ по переменным $t, c_{1}^{(m)}, \ldots, c_{n-1}^{(m)}$ вытекает голоморфность (а значит, и непрерьвность) производных в (4.2). Отсюда вьполнение условий (4.2) в точке $z_{0}$ влечет их выполнение в некоторой окрестности $O_{m}\left(z_{0}\right) \subset \widetilde{D}$. Обозначим через $U\left(z_{0}\right)$ пересечение окрестностей: $U\left(z_{0}\right)=\bigcap_{m=1}^{n} O_{m}\left(z_{0}\right)$. Окрестность $U\left(z_{0}\right)$ любой из точек $z_{0}$ плотного множества $F$ может быть выбрана в качестве подобласти в $D$, в которой степени всех полиномов $R_{i}^{(m)}(t)$ и $f_{m}(t)$ постоянны. Теорема 5 доказана.

5. Доказательство теоремы 6. Теорема 6 содержит оценку для размерности линейного пространства $V(N)$ голоморфных решений системы уравнений (3.4). Выберем некоторую точку $z_{0} \in D$. Без ограничения общности можем считать $z_{0}=0$. В силу линейной независимости векторов $\boldsymbol{X}_{1}, \ldots, \boldsymbol{X}_{n}$ в каждой точке $D$ (см. определение 2$)$ мы можем выбрать координаты $z_{1}, \ldots, z_{1}$ в окрестности точки $z_{0}=0$ так, что в самой точке $z=0$ векторы $\boldsymbol{X}_{1}, \ldots, \boldsymbol{X}_{n}$ будут направлены вдоль осей координат. Этого можно добиться за счет линейной замены переменных или, другими словами, заменой базиса в $\mathbb{C}^{n}$. Тогда

$$
\boldsymbol{X}_{m}=\frac{\partial}{\partial z_{m}}+\sum_{k=1}^{n} x_{m k}(z) \frac{\partial}{\partial z_{k}}
$$

причем голоморфные по $z$ коэффициенты $x_{m k}(z)$ обращаются в нуль при $z=0$. Пусть $\varphi(z) \in V(N)$. Разложим функцию $\varphi(z) \in V(N)$ и коэффициенты $x_{m k}(z)$ в кратные ряды Тейлора с центром в точке $z=0$ :

$$
\varphi(z)=\sum_{\alpha} \varphi_{\alpha} z^{\alpha}, x_{m k}(z)=\sum_{\beta \neq 0} x_{m k \beta} z^{\beta},
$$


где $\alpha=\left(\alpha_{1}, \ldots, \alpha_{n}\right)$ и $\beta=\left(\beta_{1}, \ldots, \beta_{n}\right)$ - мультииндексы. После этого подставим разложения (5.2) в уравнения (3.4). Такая подстановка приводит к бесконечной серии линейных однородных алгебраических соотношений на коэффициенты разложения функции $\varphi(z)$.

ЛЕмма 1. Если $\varphi \in V(N)$ u $|\alpha|=\alpha_{1}+\cdots+\alpha_{n} \geqslant n N$, то в силу одного из уравнений (3.4) для коэффициента $\varphi_{\alpha}$ имеет место соотночение

$$
\varphi_{\alpha}=\sum_{|\gamma|<|\alpha|} C_{\alpha \gamma} \varphi_{\gamma}
$$

әде $C_{\alpha \gamma}$ - некоторые константы, определяемые коэффициентами в тейлоровских разложсениях функиий $x_{m k}(z)$.

ДОКАЗАТЕЛЬСТВо. Если $|\alpha|=\alpha_{1}+\cdots+\alpha_{n} \geqslant n N$, то по крайней мере одна из компонент мультииндекса $\alpha$ больше или равна $N$. Пусть для определенности $\alpha_{m} \geqslant N$. Тогда рассмотрим $m$-е уравнение (3.4). Из (5.1) и (5.2) имеем

$$
\boldsymbol{X}_{m}=\frac{\partial}{\partial z_{m}}+\sum_{k=1}^{n} \sum_{\beta \neq 0} x_{m k \beta} z^{\beta} \frac{\partial}{\partial z_{k}} .
$$

Применим оператор $\boldsymbol{X}_{m}$ к отдельному моному вида $z^{\gamma}$, где $\gamma$ - мультииндекс. Главньй член в разложении (5.4) - это оператор дифференцирования по $z_{m}$. Его применение к моному $z^{\gamma}$ понижает на единицу показатель степени $\gamma_{m}$

$$
\gamma \mapsto \widetilde{\gamma}=\left(\gamma_{1}, \ldots, \gamma_{m}-1, \ldots, \gamma_{n}\right)
$$

если $\gamma_{m} \neq 0$. Если $\gamma_{m}=0$, то моном зануляется. Остальные слагаемые в (5.4) действуют на моном $z^{\gamma}$ сходным образом

$$
x_{m k \beta} z^{\beta} \frac{\partial z^{\gamma}}{\partial z_{k}}=\gamma_{k} x_{m k \beta} z^{\tilde{\gamma}} .
$$

Если $\gamma_{k}=0$, то правая часть (5.6) равна нулю. В противном случае в правой части получается моном со следующими показателями степеней:

$$
\gamma \mapsto \widetilde{\gamma}=\left(\gamma_{1}+\beta_{1}, \ldots, \gamma_{k}-1+\beta_{k}, \ldots, \gamma_{n}+\beta_{n}\right)
$$

Обозначим через $\delta(i)=(0, \ldots, 1, \ldots, 0)$ мультииндекс с единственной ненулевой компонентой $\delta_{i}=1$ в $i$-й позиции. Тогда правило (5.5) можно записать как $\widetilde{\gamma}=\gamma-\delta(m)$, а второе правило (5.7) запишется как $\widetilde{\gamma}=\gamma-\delta(k)+\beta$. При вычислении действия $N$-й степени оператора $\boldsymbol{X}_{m}$ на моном $z^{\gamma}$ мы должны комбинировать правила (5.5) и (5.7). Поэтому

$$
\left(\boldsymbol{X}_{m}\right)^{N} \varphi=\sum_{\alpha_{m} \geqslant N} C_{\alpha} \varphi_{\alpha} z^{\alpha-N \delta(m)}+\sum_{M<N} \sum_{\gamma, \varepsilon} C_{\gamma \varepsilon}(M) \varphi_{\gamma} z^{\gamma-M \delta(m)+\varepsilon}
$$

Здесь $C_{\alpha}=\alpha_{m}\left(\alpha_{m}-1\right) \cdots\left(\alpha_{m}-N+1\right) \neq 0$, а константы $C_{\gamma \varepsilon}(M)$ определяются коэффициентами $x_{m k \beta}$ в разложении (5.4). Первая сумма в (5.8) определяется $N$-кратньм 
действием главного члена разложения (5.4). Вторая сумма содержит вклады от $M$-кратного действия главного члена и от $(N-M)$-кратного действия остальных членов разложения (5.4) в различных комбинациях.

Величина мультииндекса $\varepsilon$ в (5.8) определяется действием неглавных слагаемых разложения (5.4) в соответствии с (5.7). Заметим, что $|\widetilde{\gamma}|>|\gamma|$ в (5.7), это вытекает из $\beta \neq 0$ в (5.2) и в (5.4). Отсюда для $\varepsilon$ в (5.8) имеем

$$
\varepsilon_{1}+\cdots+\varepsilon_{n} \geqslant 0
$$

Приведем подобные слагаемые в формуле (5.8). Для этого мы должны сгруппировать мономы с одинаковьми степенями $z$. Приравняв показатели степеней $z$ в двух суммах (5.8), получаем соотношение, связывающее $\alpha$ и $\gamma$ :

$$
\gamma=\alpha-(N-M) \delta(m)-\varepsilon
$$

После приведения подобных слагаемых само соотношение (5.8) запишется так:

$$
\left(\boldsymbol{X}_{m}\right)^{N} \varphi=\sum_{\alpha_{m} \geqslant N} C_{\alpha}\left(\varphi_{\alpha}-\sum_{\gamma} C_{\alpha \gamma} \varphi_{\gamma}\right) z^{\alpha-N \delta(m)},
$$

а коэффициенты $C_{\alpha \gamma}$ во внутренней сумме по $\gamma$ вычисляются по формуле

$$
C_{\alpha \gamma}=-\frac{1}{C_{\alpha}} \sum_{M<N} \sum_{\varepsilon} C_{\gamma \varepsilon}(M)
$$

с учетом соотношения (5.10), выражающего $\gamma$ через $\alpha, M$ и $\varepsilon$. Теперь учтем $m$-е уравнение (3.4). Оно имеет вид $\left(\boldsymbol{X}_{m}\right)^{N} \varphi=0$ и означает зануление коэффициентов при всех степенях $z$ в разложении (5.11). Отсюда

$$
\varphi_{\alpha}=\sum_{\gamma} C_{\alpha \gamma} \varphi_{\gamma}
$$

Учет условия $M<N$ в (5.8) и соотношений (5.9) и (5.10) дает $|\gamma|<|\alpha|$. Поэтому в сумме (5.12) фактически присутствуют лиш слагаемые с $|\gamma|<|\alpha|$. Значит, сумма (5.12) имеет вид (5.3). Лемма 1 доказана.

Используем лемму 1 для доказательства теоремы 6. Рассмотрим отображение $\psi$, которое каждой голоморфной функции $\varphi(z) \in V(N)$ сопоставляет начальный фрагмент ее тейлоровского разложения в точке $z=0$ :

$$
\psi: \varphi \mapsto \sum_{|\alpha|<n N} \varphi_{\alpha} z^{\alpha}
$$

Ясно, что $\psi$ - это $\mathbb{C}$-линейное отображение из $V(N)$ в пространство $P(N)$ тейлоровских полиномов вида (5.13). Оно инъективно, ибо для функции $\varphi \in V(N)$ из зануления ее начального фрагмента (5.13) в силу соотношений (5.3) из леммы 1 вытекает зануление всех коэффищиентов ее тейлоровского разложения. Инъективность линейного отображения $\psi: V(N) \rightarrow P(N)$ дает $\operatorname{dim} V(N) \leqslant \operatorname{dim} P(N)$. Размерность пространства $P(N)$ конечна и совпадает с числом слагаемых в полиномах (5.13). Отсюда возникает оценка

$$
\operatorname{dim} V(N) \leqslant \operatorname{dim} P(N) \leqslant(n N)^{n},
$$


которая завершает доказательство теоремы 6. Оценка (5.14) для $\operatorname{dim} P(N)$ является очень грубой, но для наших целей ее вполне достаточно.

Авторы признательны профессорам Э. Бедфорду и С. И. Пинчуку за приглашение в Блумингтон и возможность выступить на семинаре по комплексному анализу. Авторы также признательны профессору Лей Фу (Indiana University), обнаружившему ошибку в первоначальном варианте доказательства теоремы 6. В данном тексте она устранена.

\section{СПИСОК ЦИТИРОВАННОЙ ЛИТЕРАТУРЫ}

[1] Шабат Б. В. Введение в комплексный анализ. М.: Наука, 1960.

[2] Bochner S., Martin W. T. Several Complex Variables. Princeton: Princeton Univ. Press, 1948.

[3] Sukhov A. B. On $C R$ mappings of real quadric manifolds // Michigan Math. J. 1994. V. 41. P. 143-150.

[4] Sukhov A. B. On the mapping problem for quadric Cauchy-Riemann manifolds // Indiana Univ. Math. J. 1993. V. 42. P. 27-36.

[5] Poincaré H. Les fonctions analytiques de deux variables et la representation conforme // Rend. Circ. Mat. Palermo. 1907. V. 23. P. 185-220.

[6] Tanaka N. On the pseudo-conformal geometry of hypersurfaces of the space of $n$ complex variables // J. Math. Soc. Japan. 1962. V. 14. P. 397-429.

[7] Pelles D. Proper holomorphic self-maps of the unit ball // Math. Ann. 1971. V. 190. P. 298-305.

[8] Alexander H. Holomorphic mappings from the ball and polydisc // Math. Ann. 1974. V. 209. P. 249-256.

[9] Туманов А., Хенкин Г. Локальная характеризация голоморфных автоморфизмов областей Зигеля // Функцион. анализ и прилож. 1983. Т. 17. С. 49-61.

[10] Туманов A. Конечномерность группы $C R$ автоморфизмов стандартных $C R$ многообразий и собственные голоморфные отображения областей Зигеля // Изв. АН СССР. Сер. матем. 1988. T. 52. C. 651-659.

[11] Forstnerič F. Mappings of quadric Cauchy-Riemann manifolds // Math. Ann. 1992. V. 292. P. $163-180$.

[12] Webster S. On the mapping problem for algebraic real hypersurfaces // Invent. Math. 1977. V. 43. P. 53-68.

[13] Сухов А. Б. Об алгебраичности комплексно-аналитических множеств // Матем. сб. 1993. T. 74. C. $419-426$.

[14] Sharipov R. A., Sukhov A.B. On $C R$ mappings between algebraic Cauchy-Riemann manifolds and separate algebraicity for holomorphic functions // Preprint № 95-3. Centre de Mathématiques et d'Informatique, Univ. de Provence, Laboratotoire d'Analyse, Topologie, Probabilités (Unité de Recherche Associé au CNRS 0225), 1995; e-print alg-geom@publications . math .duke . edu/9412015.

[15] Sharipov R. A., Sukhov A. B. On $C R$ mappings between algebraic Cauchy-Riemann manifolds and separate algebraicity for holomorphic functions // Trans. Amer. Math. Soc. 1996. V. 348. № 2. P. 767-780.

[16] Рид М., Саймон Б. Методы современной математической физики. М.: Мир, 1977.

[17] Колмогоров А. Н., Фомин С. В. Теория функций и функциональный анализ. М.: Наука, 1972. 